\title{
Effects of sleeve gastrectomy in leptin deficient (ob/ob) mice and diet induced obese mice
}

\author{
Robert Bachmann*, Jessica Lange, Alfred Königsrainer and Markus A Küper \\ University Hospital Tübingen, General, Visceral and Transplantation Surgery, 72076 Tübingen, Germany
}

\begin{abstract}
Background/ Aims: Vertical sleeve gastrectomy improves weight loss and metabolic complications in obese humans. The aim of the present study was to show the outcome of sleeve gastrectomy in obese and super obese mice.

Methods: In an animal study, male wild type (WT) C57BL/6 diet-induced obese (DIO) mice were used to mimic obesity, and ob/ob mice were used for leptin deficiency-induced super obesity. To investigate whether sleeve gastrectomy works in leptin-deficient mice, the two groups were compared in weight course for 7 weeks after sleeve gastrectomy.
\end{abstract}

Results: In WT mice, vertical sleeve gastrectomy induced weight loss over the initial study period followed by weight regain over the presurgery level. In leptindeficient $\mathrm{ob} / \mathrm{ob}$ mice body weight was as well clearly reduced during the first weeks with an increase thereafter to the presurgery level.

Conclusion: Those data demonstrate that ob/ob mice and DIO mice responded differently to sleeve gastrectomy. In leptin deficient super obesity restrictive sleeve gastrectomy promotes an effective weight loss procedure. Sleeve gastrectomy leads to leptin-independent changes with favourable weight course.

\section{Introduction}

The continuous rise of obesity in the world is leading to a growing burden for health care systems and individual health. Conservative methods to weight reduction are most often failing. Bariatric surgery is the most successful strategy for treating obesity. The underlying mechanisms for this success are not clearly understood.

The most widely used bariatric surgery procedures are Roux-en-Y gastric bypass (RYGB) and vertical sleeve gastrectomy (VSG) [1]. A large multicenter study showed that the vertical sleeve gastrectomy as a stand-alone procedure seems to be a safe and effective procedure for the treatment of obesity and obesity related comorbidities [2]. Previous studies showed the effectiveness of sleeve gastrectomy in regulating body weight and ameliorating diabetes mellitus type II [3]. The effects of VSG and RYGB regarding loss of body weight, fat mass and improved glucose tolerance in humans are similar. Due to the lower operative risks and the lower postoperative morbidity, VSG is increasingly preferred [4]. Beside the restrictive character, the exact mechanisms of weight reduction after sleeve gastrectomy remained unclear. Concerning this there are considerations that the removal of the gastric fundus may affect food intake and final weight loss through hormonal pathways $[5,6]$.

We have recently established a valid sleeve gastrectomy mouse model, characterized by a small gastric pouch, low mortality and supression of body weight [7]. As restriction alone can not be the explanation of the potent effects of VSG, hormonal pathways must additionally have a decisive role. Leptin and other hypothalamic hormones regulate the energy balance over command and feedback signals. Leptin and adiponectin count to the peripheral factors that are produced by adipose tissue and regulate via the hypothalamic mechanism the long-term energy status [8]. Leptin concentration correlates with body weight and leptin-resistance contributes to hyperphagia, hypometabolism and obesity. The impact of leptin-feedback in loss and controlling of weight after sleeve gastrectomy is not known.

The present study was conducted to explore mechanisms of weight reduction including the impact of leptin-deficiency in obesity and super obesity following restrictive sleeve gastrectomy.

\section{Materials and methods}

\section{Animals}

All experiments were approved by the Institutional Animal Care and Use Committee of the University Hospital Tübingen. The institutional guidelines for the care and use of laboratory animals were followed throughout the study.

The mice were housed in the small animal facility of the University hospital of Tübingen under a light dark cycle of 12 to 12 hours.

In the study design wild type male $\mathrm{C} 65 \mathrm{Bl} / 6$ mice $(\mathrm{n}=19)$ were used in diet induced obesity (DIO) group. DIO mice were generated by feeding with a high-fat diet (D12451; 60\% calories from fat, $20 \%$ proteins, $20 \%$ carbohydrates, vitamins and minerals. Research Diets, Inc., New Brunswick, NJ) and a fructose sweetened beverage (3\%) and given free access to water for 12 weeks. After surgery DIO mice were continously fed with high-fat diet.

Correspondence to: Robert Bachmann, University Hospital Tübingen, General, Visceral and Transplantation Surgery, Hoppe-Seylerstrasse 3, 72076 Tübingen, Germany, E-mail: robert.bachmann@med.uni-tuebingen.de

Key words: mice model, sleeve gastrectomy, obesity, weight reduction

Received: September 20, 2017; Accepted: November 16, 2017; Published: November 21, 2017 
Male ob/ob mice $(\mathrm{n}=16)$ in $\mathrm{C} 57 \mathrm{Bl} / 6 \mathrm{~J}$ gene background were purchased from the Charles River Laboratories (Charles River Laboratories, Research Models and Services, Germany GmbH, Sulzfeld, Germany) at 4 weeks of age and fed the regular chow diet ad libitum before and after surgery.

\section{Surgical procedures}

Prior to surgical procedures, the mice were fasted for $24 \mathrm{~h}$ with free access to water. Sleeve gastrectomy was performed in DIO mice with a body weight of about $29 \mathrm{~g}$.

In ob/ob mice surgery was performed at 12 weeks in age with a body weight of about $63 \mathrm{~g}$.

\section{Anesthesia}

Mice were maintained by inhalation anaesthesia throughout the preparation and operation, using a scavenged mask circuit to effect 1 to $4 \%$ of isoflurane (Forane, Baxter Healthcare Corporation, Deerfield, IL 60015 USA).

\section{Analgesia}

In general anesthesia, the achievement of preoperative analgesia was performed with $5 \mathrm{mg}$ per $\mathrm{kg}$ bodyweight of Carprofen (Rimadyl, Pfizer) subcutaneously administered. For postoperative analgesia, subcutaneous Carprofen was given for further 3 days in a dosage of 5 mg per kg bodyweight.

\section{Aseptic techniques}

Standard sterile procedures were followed in all operations. The shaped abdomen was prepared with 3 times skin disinfection with uncoloured alcohol solution (Softasept ${ }^{\circ}$ N B. Braun Melsungen AG, Melsungen, Germany) and on the operating field a sterile coverage with a fenestrated drape was placed. Sterile surgical gloves and instruments were used. No prophylactic or therapeutical doses of antibiotics or antifungals were administered.

\section{Sleeve gastrectomy}

After the achievement of anesthesia, analgesia and aseptic conditions with sterile coverage, an upper midline laparotomy was performed over a length of 1.5 centimeter to the xiphoid cartilage and a self-retaining retractor was placed. The liver was gently retracted and the stomach was gently exposed out of the abdominal cavity. The short gastric vessels were ligated towards the spleen using 6/0 vicryl (Ethicon, Norderstedt, Germany) and the greater omentum was dissected from the greater curvature. The tubular sleeve was created setting a vascular (bulldog) clamp on the stomach from $3 \mathrm{~mm}$ proximal the pylorus to the gastroesophageal junction (Angle of His) with always the same volume. The excluded greater curvature was dissected along the bulldog clamp aiming a resection of over $80 \%$ of gastric volume including the complete fundus. The closure of the dissected stomach was performed from the distal to the proximal end by a full-thickness running suture (5/0 monocryl, Ethicon, Norderstedt, Germany). The sutures were knotted with itself by invagination of the proximal and distal edge of the stomach. The gastric sleeve was relocated. The laparotomy was closed by interrupted sutures (3/0 Ethilon II, Ethicon, Norderstedt, Germany).

\section{Postoperative period}

After the surgical procedure the mice were placed back into the cages. For postoperative analgesia subcutaneous Carprofen was given for further 3 days in a dosage of $5 \mathrm{mg} / \mathrm{kg}$ bodyweight. On the day of operation, mice were kept without food with free access to water. For the following three days, a liquid diet with glucose syrup and free access to water was provided with subsequent transition to free high-fat diet (60\% calories from fat) in DIO mice and the regular chow diet in ob/ob mice for the following weeks, always depending on the observed health and behavior of each animal.

\section{Physiological parameters}

Body weight was measured daily in the first 2 weeks and then weekly for 7 weeks after the surgery.

\section{Euthanasia}

In general anesthesia with isofluran (Forane, Baxter) and analgesia with $5 \mathrm{mg} / \mathrm{kg}$ bodyweight subcutaneous Carprofen (Rimadyl', Pfizer), the mice were sacrificed by cardiac puncture and exsanguination 8 weeks after the surgical procedure. On mice, which died prior to 6 weeks after surgery, a necropsy was performed.

\section{Statistical analysis}

In this study, values are presented as mean + standard deviation. Graphics were done using GraphPad Prism (Version 5.0c).

\section{Results}

\section{SG resulted in weight loss in ob/ob mice and wildtype DIO mice}

\section{Initial weight}

- DIO mice: In DIO mice ( $\mathrm{n}=19)$ SG was performed at 12 weeks on HFD with body

weight around mean 28,6 g (standard deviation $\pm 1,86 \mathrm{~g}$, range 25 $\mathrm{g}$ to $31 \mathrm{~g})$.

- Ob/ob mice: In ob/ob mice ( $\mathrm{n}=16$ ) SG was performed with a mean body weight

around $62.8 \mathrm{~g}$ (standard deviation $\pm 4.4 \mathrm{~g}$, range $52 \mathrm{~g}$ to $70 \mathrm{~g}$ ).

\section{Weight course}

DIO mice: DIO mice showed a clear weight loss for the first week with an immediate weight regain thereafter (Figure 1). Most weight reduction was in the first week post-surgery with a peak of the percentual weight loss of $40 \%$ in the first postoperative week.

ob/ob mice: ob/ob mice showed an immediate and clear weight loss in the first week with a slow weight increase thereafter (Figure $2 \mathrm{a}$ and 2b). Most weight reduction was in the first week post-surgery with a peak of the percentual weight loss of $15 \%$ in the first postoperative week.

Percentage weight loss was compared between DIO and ob/ob mice at 1, 3, 5 and 7 weeks postsurgery (Figure 3). Persistent weight reduction was observed in ob/ob mice, but not in DIO mice after sleeve gastrectomy.

\section{Intraoperative complications and peri- and postoperative mortality}

\section{DIO mice}

In DIO mice intraoperative mortality was $0 \%$. No significant bleeding was seen from either gastric exposure out of the abdominal cavity, resection or closure. Perioperative mortality during the first 

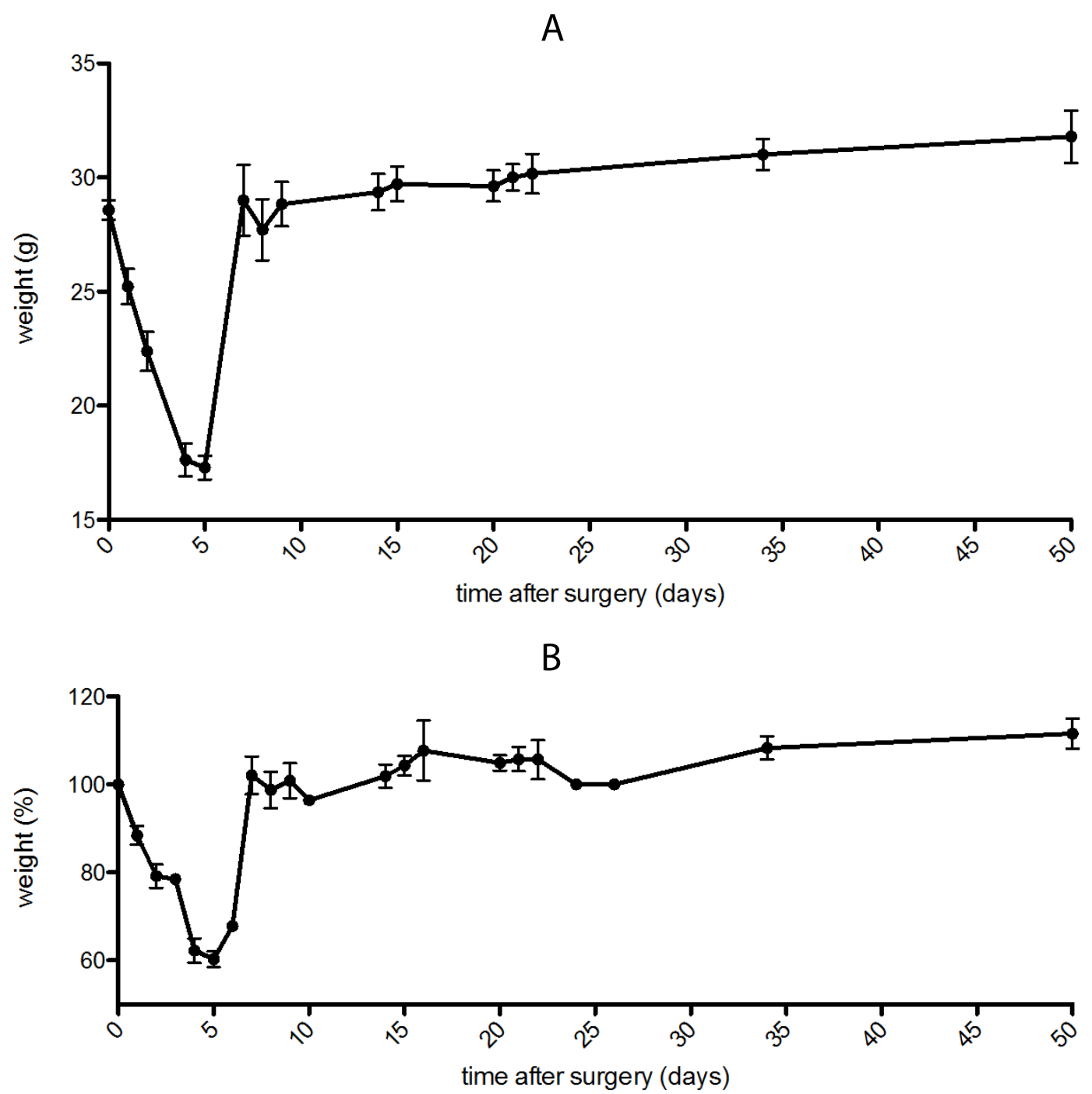

Figure 1. a) Postoperative course of absolute mean weight after sleeve gastrectomy in DIO mice. b) Percentual course of weight after sleeve gastrectomy in DIO mice.
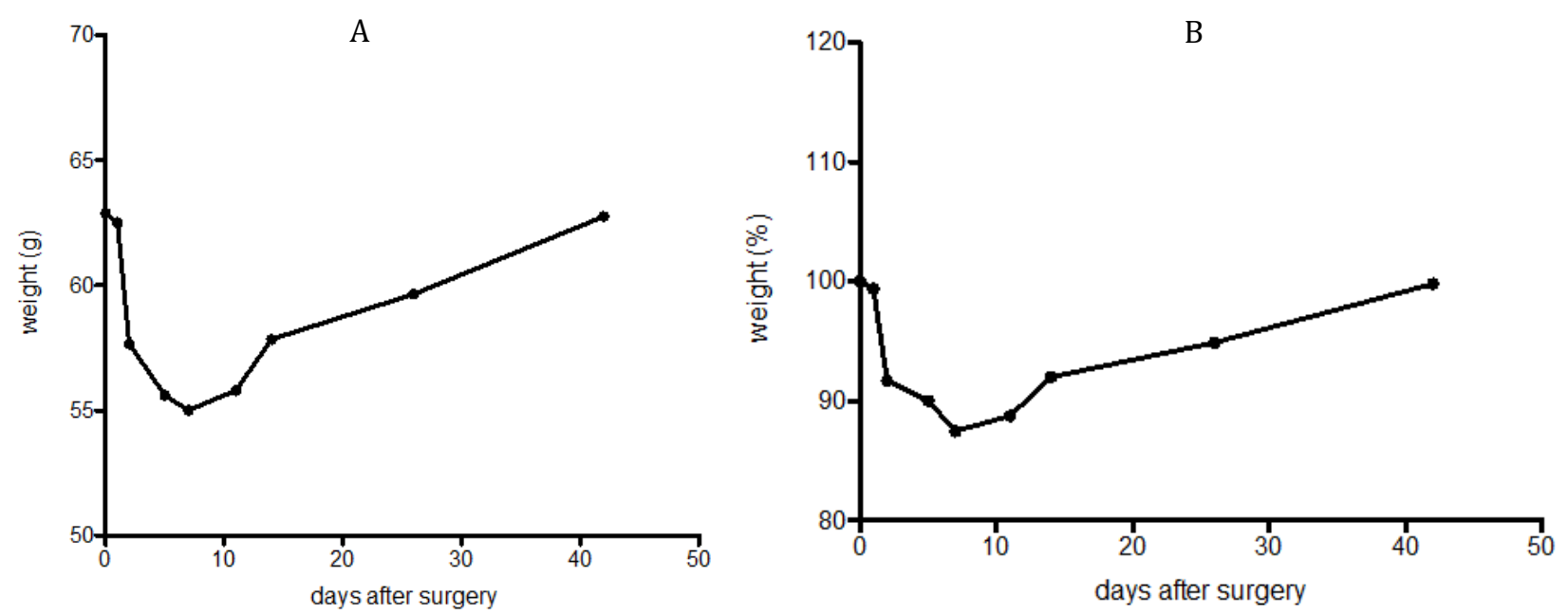

Figure 2. a) Postoperative course of mean weight after sleeve gastrectomy in ob/ob mice. b) Percentual course of weight after sleeve gastrectomy 


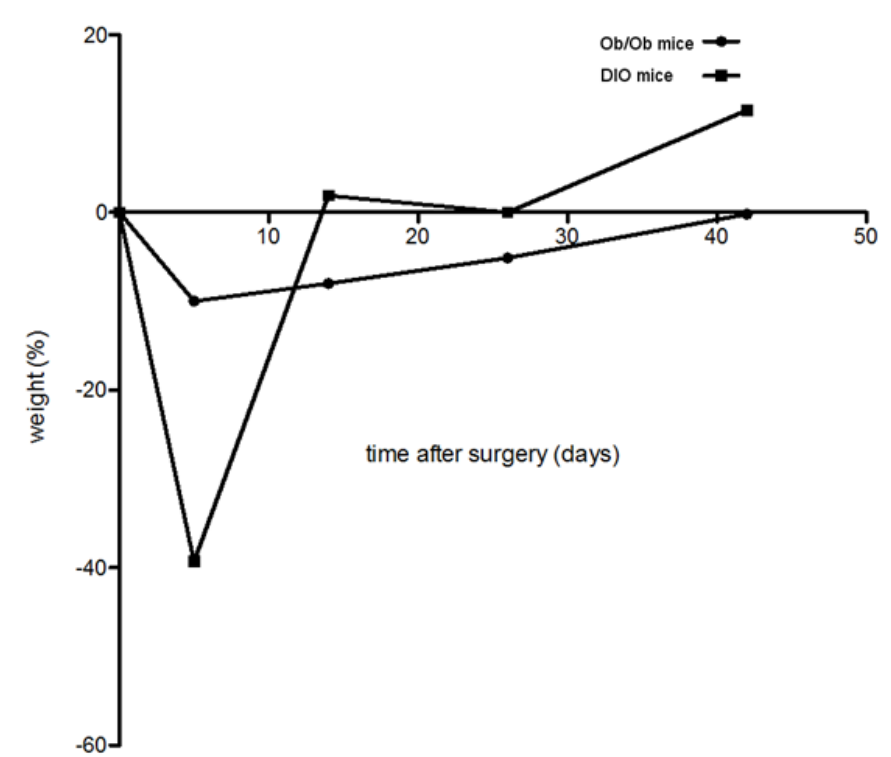

Figure 3. Comparision of the mean percentual weight course after sleeve gastrectomy. After an initial substantial mean weight loss, DIO mice ( ) showed mean weight regain surpassing their preoperative mean weight about $10 \%$. Ob/ob mice ( ) showed a moderate mean weight decrease with a slow regain in the further course without exceeding the mean preoperative weight.

week was $21 \%(n=4)$. The overall survival of the study period $(7 \mathrm{w})$ was $74 \%(\mathrm{n}=14)$.

\section{ob/ob mice}

In ob/ob mice intraoperative mortality was $0 \%$. No significant bleeding was seen from either gastric exposure out of the abdominal cavity, resection or closure. Perioperative mortality during the first week was $12.5 \%(\mathrm{n}=2)$. The overall survival of the study period $(7 \mathrm{w})$ was $75 \%(n=12)$. The overall survival of all groups was $74 \%$.

\section{Discussion}

In this manuscript, we demonstrate that the weight reducing effects of sleeve gastrectomy are preserved even in leptin-deficient $\mathrm{ob} / \mathrm{ob}$ mice. This demonstrates that the biological effects of SG are leptin-independent. We used our already published model which is characterized by close similarity to human SG surgery, including postoperative weight reduction, fast operation times and low mortality [7]. Bruinsma et al are providing another standardized procedure for mouse and rat models of Roux-en-Y gastric bypass and sleeve gastrectomy with detailed protocols [9]. Other surgical models of sleeve gastrectomy are also existing $[10,11]$.

The current study allowed us to compare DIO and ob/ob mice in sleeve gastrectomy surgery. Ob/ob mice exhibited a weight loss of $15 \%$ in the first 2 weeks post surgery with a slow increase thereafter. Nevertheless, the effect was sufficient to keep the body weight under the presurgery level during the observation period. DIO mice, however, after initial weight loss of $40 \%$ regained weight rapidly surpassing their preoperative weight early on. There are several possible explanations for these findings beginning with gastric volume restriction. The 'restrictive' hypothesis, gastric volume restriction triggers earlier satiety after SG and RYGB due to increased gastric pressure, has been challenged by recent data from both humans and rodents: Even though RYGB and VSG reduce amount of food, animals and humans still retain the capacity to consume substantially larger amounts of food and to gain significantly more body weight. Therefore, surgical induced decrease in food intake is not due to reduction of maximal food capacity, but instead reflects an intentionally decreased food consumption [12].

Furthermore, the gastrointestinal tract is not only a reabsorbing transporter, but rather an endocrine organ. This function seems -slightly modified by sleeve gastrectomy- to effectively regulates appetite, metabolism, weight and health. Leptin signaling seems not to be required for the body weight lowering effects in this SG model. Regarding weight regain Hao et al. showed in a mouse study the long term effects after RYGB in ob/ob mice. Herein the ob/ob mice regained their preoperative weight 8 weeks after RYGB. Compared to the sham operated animals RYGB led to significant difference in weight gain, but the effect was not sufficient to keep body weight below the pre-surgery levels. DIO mice, however, showed significant long term weight loss indicating that leptin-sensitivity is required for weight reducing effects of RYGB [13]. Regarding the metabolic benefits beyond weight loss,

Schneck et al. evaluated the effect of the sleeve gastrectomy on glucose, lipid metabolism, and blood pressure in a high fat diet mice model demonstrating modifications in the specific fat depots (brown vs. white adipose tissue) in response to the sleeve gastrectomy showing redistribution with prevention of ectopic fat accumulation in liver and pancreas [14]. In our leptin-deficient mice showed a significant decrease and a better outcome of weight course in the long weight trend compared to DIO mice. This result shows that SG leads to short and even long term weight loss independent from leptin. It may therefore be sufficient treatment in super obesity going beyond a pure weight decrease with respect to the results by Schneck et al.

\section{Conclusion}

Taken together, the present study shows weight reduction in DIO and leptin-deficient mice following sleeve gastrectomy. Therefore, our results suggest that leptin-sensitivity is not required for the effects of sleeve gastrectomy. However, long and short term weight course of $\mathrm{ob} / \mathrm{ob}$ mice and DIO mice were different indicating that intact leptin circuitry is at least a modulating factor in SG outcome. Future research is needed to determine whether leptin is a distinguishing mechanism between VSG and RYGB.

\section{Conflict of interest}

None of the authors has a financial interest with regard to the submitted manuscript to declare.

\section{Authorship}

RB: Planned the study. Did the animal study. Did literature search. Wrote the article. Approved the final version

JL: Did part of the animal study. Did literature search. Approved the final version.

AK: Wrote part of the article. Approved the final version.

MAK: Planned the study. Did literature search. Wrote part of the article. Approved the final version.

\section{References}

1. Eldar S, Heneghan HM, Brethauer SA, Schauer PR (2011) Bariatric surgery for treatment of obesity. Int J Obes (Lond) 35: S16-21. [Crossref]

2. Hutter MM, Schirmer BD, Jones DB, Ko CY, Cohen ME, et al. (2011) First report from the American College of Surgeons Bariatric Surgery Center Network: laparoscopic sleeve gastrectomy has morbidity and effectiveness positioned between the band and the bypass. Ann Surg 254: 410-420. [Crossref] 
3. Chang SH, Stoll CR, Song J, Varela JE, Eagon CJ, et al. (2014) The effectiveness and risks of bariatric surgery: an updated systematic review and meta-analysis, 2003-2012. JAMA Surg 149: 275-287. [Crossref]

4. Peterli R, Wölnerhanssen B, Peters T, Devaux N, Kern B, et al. (2009) Improvement in glucose metabolism after bariatric surgery: comparison of laparoscopic Roux-en-Y gastric bypass and laparoscopic sleeve gastrectomy: a prospective randomized trial. Ann Surg 250: 234-241. [Crossref]

5. Lin E, Gletsu N, Fugate K, McClusky D, Gu LH, et al. (2004) The effects of gastric surgery on systemic ghrelin levels in the morbidly obese. Arch Surg 139: 780-784. [Crossref]

6. Wang Y, Liu J (2009) Plasma ghrelin modulation in gastric band operation and sleeve gastrectomy. Obes Surg 19: 357-362. [Crossref]

7. Bachmann R, Meile T, Lange J, Widmayer P, Königsrainer A, Küper MA (2013) Surgical technique of a vertical sleeve gastrectomy in mice. J Invest Surg 26:261-265. [Crossref]

8. Angelopoulos N, Goula A, Tolis G (2005) Current knowledge in the neurophysiologic modulation of obesity. Metabolism 54: 1202-1217. [Crossref]
9. Bruinsma BG, Uygun K, Yarmush ML, Saeidi N (2015) Surgical models of Roux-en-Y gastric bypass surgery and sleeve gastrectomy in rats and mice. Nat Protoc 10: 495507. [Crossref]

10. Schlager A, Khalaileh A, Mintz Y, Abu Gazala M, Globerman A, et al. (2011) A mouse model for sleeve gastrectomy: applications for diabetes research. Microsurgery 31: 6671. [Crossref]

11. Yin DP, Gao Q, Ma LL, Yan W, Williams PE, et al. (2011) Assessment of different bariatric surgeries in the treatment of obesity and insulin resistance in mice. Ann Surg 254: 73-82. [Crossref]

12. Mumphrey MB, Hao Z, Townsend RL, Patterson LM, Morrison CD, et al. (2014) Reversible hyperphagia and obesity in rats with gastric bypass by central MC3/4R blockade. Obesity (Silver Spring) 22: 1847-1853. [Crossref]

13. Hao Z, Münzberg H, Rezai-Zadeh K, Keenan M, Coulon D, et al. (2015) Leptin deficient ob/ob mice and diet-induced obese mice responded differently to Roux-en-Y bypass surgery. Int J Obes (Lond) 39: 798-805. [Crossref]

14. Schneck AS, Iannelli A, Patouraux S, Rousseau D, Bonnafous S, et al. (2014) Effects of sleeve gastrectomy in high fat diet-induced obese mice: respective role of reduced caloric intake, white adipose tissue inflammation and changes in adipose tissue and ectopic fat depots. Surg Endosc 28: 592-602. [Crossref]

Copyright: (C2017 Bachmann R. This is an open-access article distributed under the terms of the Creative Commons Attribution License, which permits unrestricted use, distribution, and reproduction in any medium, provided the original author and source are credited. 